\section{Deutsches Register}

\author{
Loránd-Levente Pálfi*
}

\section{A}

accomodate dicere 23131

Adäquatheit 3 161-176, 161 (Def)

Äquivalenz 3 161-176, 163 (Def),

10 110, 3350 (Def)

der Begriff 3 164-169

vs Heterovalenz 3515

Kriterien 3 169-175

Text- 3163 (Def)

vs Wort- $3162 \mathrm{f}$

Äußerungsverben

Dänisch vs Deutsch 9 74-79

agapé 16158

Agricola, Erhard

Lexikographie 3145

Ahmad, Khurshid

Fachsprachenforschung 22176

Aktiv-passiv-Theorie 37 139f

Allgemeinsprache 28215 (Def)

Allori, P.E.

Fachsprachenforschung 22178

Alltagssprache 28215 (Def)

Alston, William

Bedeutungstheorie 2974

Altniederländisch

Phonologie und Morphologie 10

129-131

Andersen, Hanne L.

Bedeutung 2915

Anglophonie

in den Wissenschaften 16147
Anscombre, Jean-Claude

Bedeutung eines Wortes 970

Antisprichwörter 3530

Antonymie 18 137f

Antos, Gert

Kommunikation $872 \mathrm{f}$

Applikabilität 16 151f

aptum 23131

Aquin, Thomas von

Fachlichkeit 16160

Archisememe 6116

areté $\mathbf{1 6} 120 \mathrm{f}$

Ariel, M.

referentielle Ausdrücke 1491

Zugänglichkeitsskala 14101

Zugänglichkeitstheorie 14 91-94

artes $\mathbf{1 6} 123 \mathrm{f}$

artes incertae $\mathbf{1 6} 125$

artes liberales 16 124f, 159

artes mechanicae $\mathbf{1 6}$ 124, 159

ASCII 28224

Attribute

deutsche

Wiedergabe in norwegischen

Übersetzungen 33 89-112

Auður Hauksdóttir

dänisch-isländische Phraseologie 3695

Aufklärung 16125

Ausbildung 16123

Ausgangstext 33 71ff

Analyse

übersetzungsrelevante 33 49f

Elemente

Hierarchisierung 33 49f

Selektion 3349

Sprachnorm 3352

\footnotetext{
* Loránd-Levente Pálfi

Zentrum für Lexikographie

Wirtschaftsuniversität von Aarhus, Universität von Aarhus

Fuglesangs Allé 4

DK-8210 Aarhus $V$

llp@asb.dk
}

Hermes - Journal of Language and Communication Studies no 39-2007 
Aussagen 2988

Austrophraseologismen 35270

\section{B}

Bacon, Francis

wiederhol- und nachprüfbare

Experimente 16157

Bakker, Peter

Bedeutung $2916 \mathrm{f}$

Bally, Charles

Phraseologie $3520 \mathrm{ff}$

Barz, Irmhild

Partizipien 37142

Wörterbücher

ein- und zweisprachige 37

$139 \mathrm{ff}$

Bauer, Heinrich

es 25147

Baumann, Klaus-Dieter

Fachsprachenforschung 22 178f

integrative Fachtextlinguistik 18 203-205

Beaugrande, Robert A. de Primär- und Sekundärkonzepte 14104

Proformen 11 108f

Bedeutung

der Begriff 33 74f

empirische Grundlage 29 14-20

holistische Konzeption von 2982

Bedeutungsbeziehungen 18 135-145

elektronische Abbildung von $\mathbf{1 8}$

149-151

grundstrukturelle 18137

monodirektionale 18137

multidirektionale 18140

siehe auch Antonymie;

Feldbeziehungen; Synonymie

Bedeutungstheorie 29 73-103, 3351

Gebrauchstheorie der Bedeutung 29 79-87

nicht-korrelationistische 29 75-77

Quinesche 2995

realistische 2995 und Semantik 29 93-99

Status der 29 77-79

Begriffsbeziehungen 18 34-36, 34f begriffliche Strukturierungen $\mathbf{1 8}$

33-49

begriffliche Überschneidung 28212

logische Beziehung 1835

partitive Beziehung 1835

systematische Begriffsrepräsenta-

tion $1846 \mathrm{f}$

Unterteilung $1835 \mathrm{f}$

siehe auch Terminologie

Beier, Rudolf

Typologisierung von Textsorten

2361

Bekisheva, E.

Fachsprachenforschung 22 180f

Belegsammlungen

Übertragung in Datenbanken 5101

Benes, Eduard

die Textsorte Lexikon 2351

Berdychowska, Zofia

Wirtschaftskommunikation in

Osteuropa $29233 \mathrm{f}$

Bergenholtz, Henning

Duden 855

Lemmaselektion $37143 \mathrm{f}$

Lexikographie ein- und zweisprachige 37 139ff

vs Lexikologie $2918 \mathrm{f}$

die moderne lexikographische

Funktionslehre

vs die allgemeine Theorie der

Lexikographie 38 141f

sprachliches Feld 18131

Berger, Hugo

Unternehmenskultur 29236

Berger, Klaus

Bibelübersetzung $\mathbf{3 3} 72$

Bertels, John

Wörterbücher 7 30f, 42, 46

Bevingede Ord og Aforismer (Pol)

$35243 \mathrm{ff}$

Bevingede Ord (Vogel-Jørgensen)

35243 
BGB 28215

Biadun-Grabarek, Hanna

Wortstellung im Deutschen vs

Polnischen 879

Bibelübersetzung $\mathbf{3 3} 72$

Biere, Bernd-Ulrich

Textoptimierung 16142

Bildung 16123

Bilut, Zofia

Übersetzung 881

Bisle-Müller, $\mathrm{H}$.

Referenzprobleme 14102

Bjørge, A.K.

Fachsprachenforschung 22180

Bloomfield, Leonard

Bedeutung eines Zeichens 2977

Blum-Kulka, S.

Übersetzung vs Original 33 91f

Bolten, Jürgen

Kulturforschung 29226

Bondzio, Wilhelm

Valenz 17182

Präpositionen und Konjunktionen 17208

Bossong, Georg

Wissenschaft 16157

Bovier de Fontenelle, Bernard Le dialogische Didaktik 16143

Braunmüller, Kurt

skandinavistische Linguistik 24

181-184

Bremmer, Rolf $\mathrm{H}$.

Altniederländisch

Phonologie und Morphologie

10 129-131

Broich, Ulrich

Intertextualität 16153

Brons-Albert, Ruth

Kommunikationstraining $873 \mathrm{f}$

Brünner, Gisela

Kommunikationstypen 8 71f

Wirtschaftskommunikation 29219

Budin, Gerhard

Wissensorganisation und Termi-

nologie 22 153-158
Büchel, Georg

computergestützte Lexikographie

28224

Büchmann, Der neue 35 243ff

Bürgerliches Gesetzesbuch $\rightarrow$ BGB

Buhofer, Annelies $\rightarrow$ Häcki Buho-

fer, Annelies

Bungarten, Theo

interkulturelle Marketingkommu-

nikation $29223 \mathrm{f}$

Bibliographie 29241

Kulturforschung 29226

Burch, Thomas

TEI-Richtlinien 28 230f

Burger, Harald

Phraseologie 35 18f, 24

Terminologie 3528

pragmatische Idiome 3532

pragmatische Phraseologismen 35 32

Typologie der Modifikationen 3534

Bus-Lauer, I.-A.

Fachsprachenforschung 22180

Buscha, Joachim

Subjunktoren 17 201-203

Business-Etikette

in Europa 34 285-292

\section{C}

caritas 16158

Cherubim, D.

Textsorten 239

Chronizität 16151

Cicero

körperliches Ausdrucksverhalten

23129

Rhetorik $23127 \mathrm{f}$

der Vortrag 23129

Clae, M.-T.

interkulturelle Kommunikation

22185

collocations

vs Kollokationen 3525

COLTERM 1848 
communicative clues 10111

Compulexis 12218

Computerlexikographie 5 97ff, 28

223-235, 36 96, 38138

Coulmas, Florian

Phraseologie 3518

Routineformeln 3532

Crößmann, Helga

Subjunktoren 17201

\section{D}

Dänisch

Äußerungsverben

vs Deutsch 9 74-79

gesprochene Sprache

vs Deutsch 23 233-238

Modalpartikeln 28 135-153

Phraseographie

dänisch-deutsche 3695

Phraseologie 3695

dänisch-deutsche 3695

dänisch-isländische 3695

Sønderjysk $24183 \mathrm{f}$

Südschleswigdänisch 24182

Dänische Gesellschaft für Phraseo-

logie $\rightarrow$ DANFRAS

$\mathrm{DaF} \rightarrow$ Deutsch (als Fremdsprache)

Dahl, Trine

Fachsprachenforschung 22180

DANFRAS 3511

Danilenko, Valerija Petrovna terminologische Sphären 6166

Dansk-engelsk ordbog (Gyld) $6117 \mathrm{ff}$

Dansk-engelsk ordbog (V/B) $6118 \mathrm{ff}$

Dansk-fransk ordbog (B/H) 6 118ff

Dansk-fransk ordbog (Gyld) 6 118ff

Dansk-tysk EDB ordbog

Rezension 24 196-205

Dansk-tysk ordbog (Gyld) 6 117ff

DANTERM-Klassifikation 18 40f

Dascal, Marcelo

Rechtsverständlichkeit 29135

Debski, Antoni
Lernerwörterbücher und Grammatik 878

Definiendum 23 46-49

Definiens 23 46-49

Definitionen

lexikographische 3144

Definitor 23 46-49

deklarative Sätze 28 145-147, 150f

Deklinationsart 794

Deklinationsgrad 794

Deleen, Carl

Wörterbücher 7 34, 41

Dependenzgrammatik 24185

vs Konstituentenstrukturgram-

matik $24185 \mathrm{f}$

Syntax 24 185-189

Deppermann, Arnulf

Gesprächsanalyse 26 170-172

Derivationen

phraseologische 35 83-94

Substitution und Reduktion 35

89-94

Descartes, René

Fachprobleme der Philosophie 16

142

Deskriptoren 6 170f

Deth, Jean-Pierre van

GAL 871

Deutsch

Äußerungsverben vs Dänisch 9 74-79

als Fremdsprache 8 80f, 16147

Deutschgrammatiken für Aus-

länder 17 123-134

Unterrichtsmaterial für

Deutschlerner 17 169-180, 23

244-250

Gerichtsurteile

vs Italienisch 26 176-184

gesprochene Sprache

vs Dänisch 23 233-238

Modalpartikel 28 135-153

nationale Varianten der Standard-

sprache 3536

Phraseographie 
dänisch-deutsche 3695

Phraseologie

dänisch-deutsche 3695

deutsch-isländische 3695

Satzstruktur 24 206-209

Syntax dependentiell 24 185-189

Deutsches Patent- und Markenamt

3643

Deutsches Wörterbuch (Grimm)

Neubearbeitung

vs Ordbog over det danske

Sprog 12 218, 220

vs Oxford English Dictionary

12218,220

Deutsches Wörterbuch (WAHRIG)

vs DUW $6140 \mathrm{ff}$

Didaktik

dialogische 16143

Diehl, Nina

deutsch-russische Geschäftskontakte

29234

Dirven, René

Wirtschaftskommunikation interkulturelle Aspekte $29227 \mathrm{f}$

Dittmar, Norbert

Deutsch als Fremdsprache 880

Divergenz

vs Konvergenz 3515

Dobrovol'skij, Dmitrij

Lexikographie und Phraseographie 36109

Phraseologie 35 20, 78

-Forschung

sowjetische 3524

Phraseologismen

Äquivalenz von 36 110, 112

Auswahlkriterien für zweisprachige Wörterbücher 3699

Doctima 3642

Dokument 16163

Dolmetschen

Diskursmodelle 14 90f

dolmetschrelevante Parameter 19

$285 f$

Fremdbestimmtheit 1490
Persönlichkeitsuntersuchungen an

Dolmetschern $19286 \mathrm{f}$

Qualität der Dolmetschleistung

$19283 \mathrm{f}$

Simultan- 11 107-115, 14 89-109, 272-275

als Gegenstand der interdisziplinären Forschung 19 282-288

zur Geschichte 19283

und kognitionspsychologische

Forschung 19285

und neurophysiologische Forschung $19287 \mathrm{f}$

pronominale Wiederaufnahme

11 109-115

Verzögerungsstrategie 11 113f

Wiederholung des Ausgangs-

diskurses 3377

Zugänglichkeitsmarkierer 14 91-

94, $98 \mathrm{ff}$

Unterschiede in der Anwen-

dung 14 96-108

DPMA 3643

Drehsatz 11113

Dressler, Wolfgang U.

Primär- und Sekundärkonzepte

14104

Proformen 11 108f

Drosdowski, Günther

Duden: Deutsches Universalwörterbuch 6 139-158

Droysen, Johann Gustav die hellenistische Epoche 16157

Duden

Inkonsekvenzen in den Duden-

Bänden 8 55-69

Duden: Das große Wörterbuch der deutschen Sprache 6139

Duden: Deutsches Universalwörterbuch

Anstößiges im 6 154-157

Grammatik im 6 141-143

Rezension vom 6 139-158

Wortbildungselemente 6 144f

Duden: Richtiges und gutes 
Deutsch 8 56ff

Duekilde, Anne

Ordbog over det danske Sprog.

Supplement 12218

Dummett, Michael

realistische Bedeutungstheorien 2995

\section{E}

Eckardt, Birgit

Rechtsverständlichkeit 29108

Egerer-Möslein, Kurt

Konjunktoren, Präpositionen und

Subjunktoren 17208

Eigenkünste 16124

Eisenberg, Peter

Subjunktoren 17203

Eismann, Wolfgang

Äquivalenz von Phraseologismen 36110

elexico 37134

elocutio 23 132, 138

Engberg, Jan

Konventionen von Fachtextsorten

22 158-160

Engel, Ulrich

Subjunktionen 17200

Subjunktoren 17201

Valenz 17208

Engerer, Volkmar

Phasenverben 2919

ennoia $16120 \mathrm{f}$

Entscheidungsfrage 28 142-145, 151f

epistéme 16 119-121

der aristotelische epistéme-

Begriff 16157

Ergänzungsfrage 28 140-142

Erklärung 16143

Erklärungsrichtungen

anaphorische 16164

kataphorische 16164

EUROPHRAS 3519

exklamatorische Sätze 28 148f

Expansion 23 54-57, 58-60
Expertentum/Spezialistentum 16138

Explizitheit 3392

\section{$\mathbf{F}$}

Fabricius-Hansen, C.

Substantivrahmen 2225

Fach 756

Applikabilität 16 151f

der Begriff 16 127-130

Chronizität 16151

Expertentum/Spezialistentum 16

138

Fachgebundenheit 16 149f

fachliche Partikularisierung 16138

Fachlichkeit 16 160f

und Fachsprachlichkeit

als forschungsrelevante Größe

16 149-155

als mentalitätsgeschichtliche

Größe 16 118-127

als soziale Führungsgröße 16

127-148

als Zukunftsgröße 16 155f

Fachwissen 1842

Interdisziplinarität 16 153f

Kontrastivität 16153

Medialität 16 150f

Modell fachlicher Konstellatio-

nen im Kommunikationsprozess

16134

Sachbezogenheit 16 149f

Systematizität 16150

siehe auch artes incertae; artes

liberales; artes mechanicae;

Eigenkünste; freie Künste;

Quadrivium; scientia; Semiotizi-

tät; Sophía; téchnai enkyklioi;

téchne; Trivium; verbotene

Künste

Fachkommunikation 16 117-166,

22 144, 26 177-179, 177 (Def)

Definition und Typologisierung

von 26 177-179

externe 16130 
fachkommunikative Hermeneutik 16146

fachkommunikative Semiotik 16 152

vs Fachsprache 16150

interne vs innerfachliche 16130

Kommunikationsbarrieren 16

137-139

zwischen Fachmann und Laie

29 142-145

im Ehescheidungsrecht 29

141-161

Fachlexikographie 4 67-82, 82

(Fig), 7 53-68

Abgrenzung des Gegenstandes $730 \mathrm{f}$

Bedeutungsbeziehungen 18 127-152

Forschungsstand und Entwick-

lung $755 \mathrm{f}$

Materialerfassung 7 31-33

Quellensprache/Ordnungssprache

473

vs Terminographie 6 167f

Textkorpora 733

Theorie und Praksis 7 29-50

Zielsprache/Zuordnungssprache 4

73

siehe auch Lexikographie;

Sachlexikographie

Fachsprache 16 117-166, 22 144, 155

Beschreibung einer 756

Definition des Begriffs Gentechnik

6127

11th European Symposium on LSP 22 175-189

fachsprachliche Analyse 757

Fachsprachlichkeit

und Fachlichkeit

als forschungsrelevante Größe

16 149-155

als mentalitätsgeschichtliche

Größe 16 118-127

als soziale Führungsgröße 16

127-148

als Zukunftsgröße 16 155f

graduelle Stufung der $\mathbf{1 6}$ 135f
Forschung 7 55, 16 150-156, 162, 22 153, 175-189

Standortbestimmung und

Weiterentwicklung $22188 \mathrm{f}$

Standortdefinition 22176

und Übersetzung 22183

vs Gemeinsprache 16 135f

juristische(n)

vs Allgemeinsprache 28 211-221

Experten-Laien-Kommunikation

36 55-63

Polysemie in der 28 211-221

vs Sachsprache 6129

vs Terminologie 6162

wissenschaftliches Schreiben 22

184

siehe auch Wirtschaftssprache

Fachsprachen-Hermeneutik 16146

Fachsprachensemiotik 16152

Fachtexte(n)

Fachtextsorten $893 \mathrm{f}$

Analyse deutscher und däni-

scher Gerichtsurteile 22 158-160

Konventionen der 22 158-160

technische 23 41-62

Stellenwert des Adressaten 23

41-49

Forschung 26 176-184

juristische

Notwendigkeit der Komplexität

3661

kontrastive Fachtextpragmatik 26

176-184

das Verstehen der 20 259-264

Fachtextlinguistik 13 326-330

integrative 18 203-205

Fachwörterbücher(n) 4 67ff, 7 29-50

Beispiele in $743 \mathrm{f}$

Belege in $743 \mathrm{f}$

Benutzung 4 68-78, 81 (Fig)

bidirektionale $748 \mathrm{f}$

eigene Bildungen in 7 47f

ein- vs zweisprachige 743

enzyklopädische Glossen 7 43, 49 
enzyklopädische Informationen in 7 41-44, 21 205-213

falsche Freunde in 747

Grammatik in $744 \mathrm{f}$

historischer Wortschatz in $738 \mathrm{f}$

als Kontrollinstanz 736

mehrsprachige 7 48-50

Mehrworttermini in 737

Metasprache in $745 \mathrm{f}$

multidirektionale 7 48-50

Neologismen in 738

Phraseologie in $740 \mathrm{f}$

Prinzip der Verifizierung 747

semantische Informationen in $\mathbf{2 1}$

205-213

Stichwortauswahl in 7 33-40

unidirektionale $748 \mathrm{f}$

Vollständigkeit in 7 34-36

Wortbildung in 7 35-38

Zuverlässigkeit der Angaben in 7 46-48

Falkesgaard, Bendt

Unterrichtsmaterial für Deutsch-

lerner 23 244-250

Farø, Ken

Äquivalenz von Phraseologismen

36112

Kulturspezifik in der Phraseologie 36112

Feilke, Helmuth idiomatische Prägung 3527

Feldbeziehungen 18 143-149 siehe auch Bedeutungsbeziehungen

Felder 18 131-135

Handlungsfeld 18 147-149

Sachfeld $18146 f$

semantisches Feld 18 145f

Feldprinzip 18 253, 22 20-24

Festigkeit, Theorie der 3523

fides 16158

Fiehler, Reinhard

Kommunikationstraining $873 \mathrm{f}$

Filipenko, Tatjana sowjetische Phraseologieforschung 3524
Fillmore, Charles

frames $\mathbf{3 3} 20$

and scenes 3316

Scenes and Frames-Theory 3319

Theorie der Bedeutung 3351

Fleischer, Wolfgang

Phraseologie 35 18, 24

in Wörterbüchern 36102

Fluck, Hans-Rüdiger

Kommunikationsbarrieren

zwischen Fachmann und Laie

29143

Fonsmark, Henning

Textkorpora $6125 \mathrm{f}$

Forstreuter, Eike

Konjunktoren, Präpositionen und

Subjunktoren 17208

Fortschritt

der Begriff 16 122, 126, 158

Fournier, Johannes

TEI-Richtlinien 28 230f

Fragen

Definitions- 23132

Entscheidungs- 28 142-145, $151 \mathrm{f}$

Ergänzungs- 28 140-142

Rechts- 23132

Verfahrens- 23132

Vermutungs- 23132

Frames 18 131-135, 33 12, 15-41,

$51-53,65 \mathrm{f}$

cultural 33 28, 36-38, 37 (Def)

domain 3327 (Def), 28

institutional 3338

linguistic 33 28, 38 (Def), $38 \mathrm{f}$

self-concept 3327 (Def), 28

situational 3327 (Def), 33-36, 33

(Def)

social 3327 (Def), 36 (Def)

text type 3328

Typologie 33 12, 27-39

Manuel de Vega 3327

visual 3327 (Def), 28-32, 28 (Def)

wesentliche Merkmale 33 21-24

Fredsted, Elin

gesprochene Sprache 
Dänisch vs Deutsch 23 233-238

freie Künste 16124

Fremdsprachenunterricht 17 169-180, 23 244-250

Fügungspotenz 17181

\section{G}

geflügelte Worte 35242 (Def)

genuine 35 244-246

Lexikalisierung $35248 \mathrm{f}$

und Phraseologie 35250

wissenschaftlich betrachtet 35

239-253

vs Zitate 35 245-248

Zitathaftigkeit 35 246-248

Geißner, H.K.

Kommunikationsbarrieren 29 239f

Wirtschaftskommunikation $29222 \mathrm{f}$

Gemeinsprache 28215 (Def)

vs Fachsprache 16 135f

Generative Syntax 24186

Gerichtsurteile(n)

analyse von 9 71-76

deutsche vs italienische 26 176-184

sprachliche Merkmale in 9 65-81

Gesprächsanalyse 22 133-148, 26

170-172

mündliche Verhandlungen im

Gericht 22 133-148

und Terminologie 22 140-144

gesprochene Sprache

Dänisch vs Deutsch 23 233-238

Ghenghea, V.A.

Fachsprachenforschung 22180

Ghostwriter 875

Gläser, Rosemarie

Fachtextsorten im Englischen 8

93-98

medizinische Dialoge in Roma-

nen 22183

Metakommunikation 898

Glinz, Hans

Textsorten 240

Gloning, Thomas
Goethes Wortschatz und das

Goethe-Wörterbuch 28 229f

Gniffke-Hubrig, Christa

Textsorten 239

Göpferich, Susanne

Lexikon als Textsorte betrachtet

2351

technical writing 2342

Textsorten in Naturwissenschaf-

ten und Technik 16228

Goethe, J.W. von

das berühmte Götz-Zitat 22141

Index zu Goethes Wahlverwandt-

schaften 10 29-38

Verskonkordanz zu Goethes

Faust 10 34, 36

Goethe-Wörterbuch 1035

Graesser, A.C.

Scripts 3333

Grammatik

Anwendungsbereich und

Adressatenkreis 17 126-128

Dependenz- 24185

vs Konstituentenstruktur- $24185 f$

Syntax 24 185-189

deutsche 7 87-102

Korpusbeispiele 795

Deutschgrammatiken für Aus-

länder 17 123-134, 23 244-250

der Duden-Grammatik 8 57ff

Präpositionen in 8 57ff

Informantenbefragungen zur Auf-

findung von grammatischen

Regeln 25 143-157

Konstituentenstruktur-

vs Dependenz- 24 185f

linguistische vs didaktische 17 173f

die Munksgaard-Reihe 7 87-89, 100

Phrasenstruktur- 24187

Produktions- vs Rezeptions- 17 132f

das rezeptionsgrammatische

Modell 18 252f, 22 13-46

rezeptive $2215 f$

Text- $17133 \mathrm{f}$

Typologie 17 123-126 
Unterrichtsmaterial für Deutschlerner 17 169-180, 23 244-250

Gréciano, Gertrud

Phraseologie 35 19f

Grinev, S.V.

Fachsprachenforschung 22180

Gülich, Elisabeth

Phraseologie auf Text-Ebene 35 30f

Günther, Johann

Wirtschaftskommunikation 29236

Güteverhandlungen 22 140-148

Gutt, Ernst-August

communicative clues 10111

kommunikative Signale 969

Übersetzung 9 68f

\section{H}

Häcki Buhofer, Annelies

Phraseologie 35 18, 24

Erwerb der 3535

als dritte Gliederungsebene der

Sprache 3532

Terminologie 3528

pragmatische Phraseologismen 35

32

Typologie der Modifikationen 3534

Häusermann, Jürg

freie Wortverbindungen und

Phraseologismen 36 113f

Phraseologie 36110

Hamburger Gruppe 16140

Handwörterbuch der deutschen

Gegenwartssprache 3 148ff

Harras, Gisela

brisante Wörter 16130

Haß, Ulrike

brisante Wörter 16130

elexico $37134 \mathrm{f}$

Mikrostruktur im Hypertext-

wörterbuch $28227 \mathrm{f}$

Haß-Zumkehr, U. $\rightarrow$ Haß, Ulrike

Hattenhauer, Hans

Dilemma der Interdisziplinarität

$29141 f$
Hauptsatzkonjunktionen 17203

Hegstrom, T.G.

Wirtschaftskommunikation $29234 \mathrm{f}$

Heiden, Tanja

frame-Typologie 3312

Heilmann, Axel

deutsche Satzstruktur 24 206-209

Heinemann, Wolfgang

Situationstypen 23236

Heinrich, Carl

Wörterbücher 744

Heinsius, Theodor

es 25147

Heisenberg, Werner

Wissensordnung 1834

hellenistische Epoche, die 16157

Henke-Brown, Käthe

Textsorten 3531

Henne, $\mathrm{H}$.

Textsorten 239

Herbig, Albert F.

Wirtschaftskommunikation 29

222f, 234

Heringer, H.-J.

deutsche Syntax dependentiell 24

185-189

Hermeneutik

fachkommunikative 16146

Fachsprachen- 16146

fachtextuelle 16146

Herrlitz, Wolfgang

Wirtschaftskommunikation

interkulturelle Aspekte 29229

Hess, Rainer

Manierismus 3357

Hess-Lüttich, E.W.B.

interkulturelle

Wirtschaftskommunikation $29225 \mathrm{f}$

Hesse, Harald

Koordinierte Einzelobjekte 17207

Hessky, Regina

Phraseologie und Lexikographie

36103

Heterovalenz

vs Äquivalenz 3515 
Heuberger, Katharina

Wirtschaftssprache $29219 \mathrm{f}$

Hintikka, Jaakko semantische Spiele 2983

Hjelmslev, Louis

Sprachtheorie 21 206, 208

Höflichkeit 23237

Hoffmann, Lothar

Abstraktionsstufen 16162

homo faber $23121 \mathrm{f}$

homo sociologicus 23121

Homographie $28212 \mathrm{f}$

Homonymie $28212 \mathrm{f}$

Homophonie $28212 \mathrm{f}$

Hotimsky, F.

Interkulturalität 22185

Humor

der Begriff $\mathbf{3 3} 48$

Begriffserklärung 33 47f

übersetzen 33 45-67

siehe auch das Komische

Humour Studies 3346

Hyland, Ken

wissenschaftliches Schreiben 22 184

Hyperbaton $3358 \mathrm{f}$

Hyperlinks 38146 (Def)

Hypertext 28226 (Def)

-Systeme 28225

Hyvärinen, Irma

adjektivische Zusammenbildungen

37141

\section{I}

Idiomatik

der Begriff 3514

idiomatische Prägung 3527

Idiomatizität 3693

Idiome 37143

Anteil in der gesprochenen

Sprache 3533

deutsch-finnische Lexikographie

37143

pragmatische 3532 statistisch 3537

Wortgruppen- 37143

Ignatov, Marin

Wirtschaftskommunikation in

Osteuropa 29233

imperativische Sätze 28 147f

Industrielle Revolution, Dritte 16126

Industrielle Revolution, Zweite 16

126

Infinitivkonjunktionen 17194

Informantenbefragungen

zur Auffindung von grammati-

schen Regeln 25 143-157

Information

vs Wissen 22155

Informationsspaltung 33109

Ingendaay, Paul

litterarische Übersetzung

kulturspezifische Elemente 33 63f

Inhaltsstruktur (lexikographisch) 38

$140 f$

vs Vernetzungsstruktur 38148

Inklusionsmodell 23 125, 126 (Fig)

Interaktion 29126

der Begriff 29126

Ebenen und Formen 29 127-135

Leser-Text- 29 127-135

Interdisziplinarität 16 153f

Interkulturalität 16 154f, 22 176f

interkultureller Transfer 33 50-53

Interpretationsinstrumentarium

juristisches $3655 \mathrm{f}$

Intertextualität 16 153, 33 64f

Intuition $10110 \mathrm{f}$

Intuitionismus $2995 \mathrm{f}$

Isländisch

Phraseologie 3695

dänisch-isländische 3695

deutsch-isländische 3695

Sprachplanung 22186

Italienisch

Gerichtsurteile

vs deutsche 26 176-184 
J

Jacobsen, Ronald R.

Wissenssysteme 3313

Jahr, Silke

Fachtexte 20 259-264

Jakobsen, Lisbeth Falster

Kategorie der Satzglieder 878

Jakobson, Roman

die sechs Sprachfunktionen 23235

Japanisch

Sprichwörter $35272 \mathrm{f}$

Jørgensen, Poul E.

Dansk-tysk EDB ordbog 24 196-205

Jørgensen, Sven-Aage

Nationalhelden in Europa 6 200f

K

Kalverkämper, Hartwig

Fachkommunikation 26184

Fachsprachenforschung $22188 \mathrm{f}$

Gemeinsprachlichkeit und

Fachsprachlichkeit 23238

Interkulturalität 22 176ff

Kammerer, Matthias

Hyperlinks 38146 (Def)

Verweise im elektronischen Wör-

terbuch? 38143

Kanten 38 159, 163

ausgehende 38163

eingehende 38163

Third-Party- 38163

Kappel, Guido

deutsch-russische Geschäftskontakte 29234

Kasusrektion 17194

Katny, Andrzej

Bibliographie zum deutsch-

polnischen Sprachvergleich 878

Bibliographie zu Modalverben im

Englischen 881

Linguistik und Übersetzung 8 77-84

Keller, René

interkulturelle Marketingkommu-

nikation 29240
Kettenprinzip 18 253, 22 24-28

Klammerprinzip 18 252, 22 17-19

Klassifikation 6 170f

Klein, Wolfgang

Das digitale Wörterbuch der

deutschen Sprache $37134 \mathrm{f}$

Klosa, Annette

CD-ROM-Publikationen von

Wörterbüchern 28227

Koeppel, R.

Routineformeln 14105

Körpersprache

in Wirtschaftskommunikation 23

115-146

Koller, Werner

Äquivalenz von Phraseologismen

36110

Phraseologie 3518

Kollokationen

vs collocations 3525

das Komische 33 48ff

bei Eduardo Mendoza 3365

Mehrdimensionalität 33 52f

Übersetzung 3350

siehe auch Humor

Kommunikation 8 72f, 23 115-125

communicative clues $\mathbf{1 0} 111$

Ebenen 6 128f, 129 (Fig)

Experten-Laien- 29 142-145, 36

55-63

Fach- 16 117-166, 22 144, 26

177-179, 177 (Def)

Definition von 26 177-179

externe 16130

fachkommunikative Hermeneutik 16146

fachkommunikative Semiotik

16152

vs Fachsprache 16150

interne vs innerfachliche 16130

juristische 36 72f

Kommunikationsbarrieren 16

137-139

zwischen Fachmann und Laie 29 142-145, 36 55-63 
im Ehescheidungsrecht 29

$$
\text { 141-161 }
$$

Semiotik 16152

technische $16141 \mathrm{f}$

Typologisierung von 26 177-179

interkulturelle 34 285-292

Marketing-

interkulturelle $29217 \mathrm{ff}$

Bibliographie zur 29241

Sprache und Kultur in $29217 \mathrm{ff}$

und Wirtschaftskommunikation

29 219-221

Modell 29143

fachliche Konstellationen im

Kommunikationsprozess 16134

Richtungen 6129 (Fig)

Signale 969

Training $873 \mathrm{f}$

Typen 8 71f

Wirtschafts- 29 217-242

Adaption 29240

Informationstechnologien 29

236-239

interkulturelle 23 115-146

interne $29234 \mathrm{f}$

Körpersprache in 23 115-146

Lakunen 29235 (def), 235f

und Marketingkommunikation

29 219-221

in Osteuropa 29 232-234

Stereotypen $29231 \mathrm{f}$

siehe auch Nachrichtenquadrat

Kondensation 23 50-53, 58-62

Konditionalsätze 28152

Konjugationsartrektion 17196

Konjunktionen 17194

Hauptsatz- 17203

Infinitiv- 17194

Konjunktiv 793

Konjunktoren

Ausdrucksselektion 17207

vs Präpositionen 17208

Valenzeigenschaften 17 204-209

Valenzverhältnisse 17 181-209

Konstituentenstrukturgrammatik 24
185

vs Dependenzgrammatik 24 185f

Konstruktivismus

kognitive 16139

Kontrastivität 16153

Konvention 243 (Def)

Konvergenz

vs Divergenz 3515

Konversationsanalyse $\rightarrow$ Gesprächs analyse

Koop, Werner

kommunikative Barrieren $29231 \mathrm{f}$

Kopperschmidt, Josef

Rhetorik 23127

Korhonen, Jarmo

Lexikographie ein- und zweisprachige 37 139ff

Korpora $\rightarrow$ Textkorpora

Korpuslinguistik

und Phraseographie 36 97-102

Korrelate 17197

Gruppen 17197

Koskela, Merja

wissenschaftliches Schreiben 22

184

Krafft, Ulrich

Phraseologie auf Text-Ebene 35 30f

Kretzenbacher, H.L.

wissenschaftliche Kommunikation

22186

Krings, Hans P.

Anspruchslosigkeit des Berufsbildes

16163

Kristeva, Julia

Intertextualität 16153

Kriterium

der Begriff 16159

Kritik

der Begriff 16159

Kromann, H.-P.

Fachlexikographie 743

Kühn, Peter

Wörterbücher zu Phraseologismen

36117

Küper, Christoph 
Übersetzung 883

Küstner, Andreas

Koordinierte Einzelobjekte 17207

Kultur

der Begriff 23 120f

Interkulturalität 16 154f, $22176 f$

Lach- 3346

Sprach- 16156

Unternehmens- 29236

Kultureme-Didaktik 23144

Kulturforschung 29226

Kunst

bildende 23 115-125

Kunze, Claudia

GermaNet 28 232f

\section{L}

Lachkultur 3346

Lammers, Karl Christian

Österreich und Deutschland 6198

Langenscheidts Großwörterbuch

Deutsch als Fremdsprache 25 160ff

Langer, Inghard

empirisch-induktives

Verständlichkeitskonzept 16140

Laurén, Christer

Terminologie 21 181-188

Lauridsen, Ole

Unterrichtsmaterial für Deutsch-

lerner 23 244-250

Lausberg, Heinrich

Rhetorik 23127

Leech, G.

Höflichkeit 23236

Leffler, O.E.

Wörterbücher 7 31, 34, 41, 46

Lehr, Andrea

Verweise im elektronischen

Wörterbuch? 38143

Lehtonen, Jakko

Wirtschaftsethik 29240

Leibniz, Gottfried W.

die Worte 18177

Leisi, Ernst
Gebrauchstheorie 29 79, 81

Lemberg, Ingrid

Lexikographie

computergestützte $\mathbf{2 8}$ 223-235

Online- $28226 f$

Lemma

-Lücken 37143

-Selektion 37 143f

Lemmatisierung 6114

Probleme 10 34-38

Repräsentationsprobleme 1033

vs Wort 6114

Lemmabuch 6114

Lemnitzer, Lothar

Wörterbuchbenutzungsforschung 28 233f

Lenz, Werner

der unbekannte Lexikon-Redakteur 18 178f

Leppällä, Kirsi

kulturelles Wissen in der Wer-

bung $29235 \mathrm{f}$

Lernerwörterbücher(n) 25 159-168

und Grammatik 878

Routineformeln in 37143

Lesbarkeitsforschung 16139

Leser 29128

-Text-Interaktion 29 127-135

-Text-Modell 29134

Lewis, D.

Definition von Konvention 243

lexikalischen Funktionen, Theorie

der $\mathbf{3 5} 24$

Lexikalisierung $35248 \mathrm{f}$

Lexikographie 37 131-136

Aktiv-passiv-Theorie 37 139f

Angaben

der Terminus 38140

Vor- 38140

Bedeutung der Korpuslinguistik

36102

Computer- 5 97ff, 28 223-235, 36

96, 38138

Daten

Vernetzungsstrukturen 38 137-168 
XML-basierte Modellierung 38 137-168

Datenauszeichnung inhaltsorientierte 38139 layoutorientierte 38139

Datenbasis 38141

deutsch-finnische

Idiome in 37143

ein- und zweisprachige 37 139-148

Fach- 4 67-82, 82 (Fig), 7 53-68

Abgrenzung des Gegenstandes

$730 \mathrm{f}$

Bedeutungsbeziehungen 18

127-152

Forschungsstand und Entwicklung $755 f$

Materialerfassung 7 31-33

Ordnungssprache 473

Quellensprache 473

vs Terminographie $6167 \mathrm{f}$

Textkorpora 733

Theorie und Praksis 7 29-50

Zielsprache 473

Zuordnungssprache 473

die Funktionslehre 37 145-147

vs die allgemeine Theorie der

Lexikographie 38 141f

Kritik 37 145-147

die gesellschaftliche Verantwortung

18 177-199

Idiome $37146 \mathrm{f}$

Inhaltsmodellierung

konzeptuelle 38139

layoutbasierte 38139

kontemplative 35257

Lexika als Textsorte betrachtet $\mathbf{2 3}$

51

lexikographische Funktionen 37139

Kern- 37 139, $146 f$

Korrektur 37142

wissensbezogene $37146 \mathrm{f}$

Dokumentation 37146

das lexikographische Nachschla-

gewerk 21 209f

lexikographische Strukturen
Inhalts- 38 140f

vs Vernetzungsstrukturen 38148

Makro- 7 33-40

Medio-

vs Vernetzungsstrukturen 38

$145 \mathrm{f}$

Mikro- 7 40f

Vernetzungs- 38 137-168

vs Inhaltsstrukturen 38148

XML-basierte Modellierung

38 137-168

Verweis- 38137

-voraussetzungen 38143

vs Linguistik 35 256-258

linguistische 35257

maschinelle Unterstützung 5 97-109

ein modulares Modellsystem 5

103-108, 104f (Fig)

Meta-

Typen von Beiträgen 35 257f

Nutzung des Hypertextkonzepts

$28225 f$

und Ortographie 37 142f

und Phraseographie 36 96f

Rechts- 35 255ff

Sach- 755

Sprach- 755

vs Terminologie 4 75, 21 185f

Theorie und Praksis 3 145-147

transformative 35257

Typologie 21210

Vernetzungen 38 146f (Def)

bidirektionale 38 149ff, 167 (Def)

Datenbasis-externe 38 148f

Datenbasis-interne 38 148f, 151

Inhaltsstruktur-interne 38 147, 151

unidirektionale 38 149ff, 150

(Fig), 158 (Fig), 167 (Def)

Verweise 38146

Beziehungen 38147

im elektronischen Wörterbuch?

38143

Verweisvoraussetzungen 38143

siehe auch Phraseographie; Wör-

terbücher 
Lexikon-Wortformen 475 vs Text-Wortformen 3581

Liimatainen, Annikki Fachgebietsangaben in allgemeinen Wörterbüchern 37141

Lind, Olof

Wörterbücher 731

Linguistik

Fachtext- 13 326-330

integrative 18 203-205

kognitive 18 238-240

kontrastive 8 77-81

Korpus-

und Phraseographie 36 97-102

vs Lexikographie 35 256-258

linguistische Datenverarbeitung

lexikographische Problemstel-

lungen $597 \mathrm{f}$

linguistische Investition 871

skandinavistische 24 181-184

Text- 16 139, 3671

Fach- 13 326-330

integrative 18 203-205

vs Übersetzungswissenschaft 10

109

Wirtschafts- 23135

Link, E.

probleme der Lemmarepräsentation 1033

Link-Bank 38 159, 165f, 168

Links

erweiterte 38159 (Def)

Lipinski, Krzysztof

Übersetzung 8 82f

List, Pia

kommunikative Barrieren $29231 \mathrm{f}$

Lohmeier, Dieter

Schleswig-Holstein 6196

Lüger, Heinz-Helmut

Satzphraseologismen 3529

Lüsebrink, Hans-Jürgen

Wirtschaftskommunikation

Kulturunterschiede 29227

Lundqvist, Lita

Fachsprachenforschung 22 175-189
Lux, Friedemann

Textsortenforschung 238

M

Makrostruktur (lexikographisch) 7

33-40

Manierismus 3357

Marketingkommunikation

interkulturelle $29217 \mathrm{ff}$

Bibliographie zur 29241

Sprache und Kultur in 29 217ff

und Wirtschaftskommunikation

29 219-221

siehe auch Wirtschaftskommuni-

kation

Mecner, Pawel

mediale Konstruktionen

Deutsch vs Polnisch 879

Medialität 16 150f

Mediostruktur (lexikographisch)

vs Vernetzungsstruktur 38 145f

Meibauer, Jörg

kontrastive Linguistik 8 77f

Mejri, Salah

Phraseologie als dritte Gliederungsebene der Sprache 3532

Mendoza, Eduardo

El misterio de la cripta embrujada

33 53ff

mentalen Räume, Theorie der 3311

(Def)

Merk, Vincent

Wirtschaftskommunikation

interkulturelle Aspekte $29228 \mathrm{f}$

Metapherntheorie

kognitive $35268 \mathrm{f}$

Mieder, Wolfgang

Antisprichwörter 3530

Mikrostruktur (lexikographisch) $740 \mathrm{f}$

Modalpartikel

Dänisch 28 135-153

Definition der MP-Funktion 28137

denn 28140 
Deutsch 28 135-153, $136 \mathrm{f}$ etwa 28143

ja 28145

schon 28149

Modellierung 3527

Modifikationen

phraseologische 35 34f, 78ff, 83f

Modifikationsmechanismen 35

$83 \mathrm{ff}$

Typologie 3534

Möller, J.G.P.

Wörterbücher 740

Mogensen, Jens Erik

Aktiv-passiv-Theorie 37139

Ortographie und bilexikographische

Praxis $37142 \mathrm{f}$

Monitoring, linguistisches 36 55-63

Moon, Rosamund

Idiome

Anteil in der gesprochenen

Sprache 3533

statistisch 3537

lexikalische Substitution 3538

Morphologie

Altniederländisch 10 129-131

Mourier, L.

Übersetzung 22183

Müller, Carolin

Publikationsmodell in der Lexi-

kographie 28 224f

Mugdan, Joachim

Duden 855

Multimedia 28226 (Def)

Myking, Johan

Terminologie 21 181-188

Terminologieforschung 22181

\section{$\mathbf{N}$}

Nachrichtenquadrat 29143 (Fig)

Naziemkowska-Katny, M.A.

sprachliche Fehlern 879

Neubert, Gunter

Fachlexikographie 7 35f, 42

eigene Bildungen in
Fachwörterbüchern 7 47f

Textwörter vs Lexikonwörter 3581

Neutralisation 3360

Niehaus-Lohberg, E.

Wirtschaftskommunikation

interkulturelle Aspekte 29229

Nielsen, Birgit S.

deutsche Autoren in DK 6 198f

Nielsen, Sandro

Benutzungsanleitungen von

Wörterbüchern 37 141f

Nistrup-Madsen, B.

Terminologieforschung 22181

Nølke, Henning

Bedeutungstheorie 2916

Nord, Britta

Hilfsmittel beim Übersetzen $35261 \mathrm{f}$

Nord, Christiane

Bibelübersetzung 3372

Nullsubjunktoren 17200

Nuopponen, Anita

Wissensrepräsentation $18135 \mathrm{f}$

Wissensstrukturen 22182

Nussbaumer, Markus

Rechtsverständlichkeit 29108

$\mathbf{O}$

ODS $\rightarrow$ Ordbog over det danske

Sprog

Øhrgaard, Per

deutsche Geschichte 6 196-198

Entwicklung der DDR 6 199f

Öhrlander, C.N.

Wörterbücher 7 31, 34, 41, 46

Oldenburg, Hermann

Fachtextlinguistik 13 326-330

onomasiologische Prinzip, das

vs das semasiologische Prinzip 6 168

Ordbog over det danske Sprog 12 217, 223

Homogenitätsprobleme 12218

Neubearbeitung

vs Deutsches Wörterbuch 12 
218,220

vs Oxford English Dictionary

12 218, 220

Ordbog over det danske Sprog.

Supplement 1

Rezension 12 217-224

Ortographie

und bilexikographische Praxis 37

$142 \mathrm{f}$

outdoor games 2983

Oxford English Dictionary

Neubearbeitung

vs Deutsches Wörterbuch 12

218, 220

vs Ordbog over det danske

Sprog 12 218, 220

\section{$\mathbf{P}$}

Paarformeln 3527

Palm, Christine

Phraseologie 3519

Paralleltext 3365 (Def)

Parömiologie 35 267-273

vs Phraseologie 35 29f, 3693

Parsenow, Günter

Wörterbücher 746

Partikeln 17181 (Def)

Modal-

Dänisch 28 135-153

Definition der MP-Funktion 28

137

denn 28140

Deutsch 28 135-153, 136f

etwa 28143

ja 28145

schon 28149

Partikelvalenz im Deutschen $\mathbf{1 7}$ 181-209

Pascal, Blaise

Fachthemen der Religion 16142

Past-fiction 33 15-41

Patente

Allgemeines zum Patentwesen 36

42-46
Erstellungssituation 3648

linguistische Gutachten für 36 41-52

als Textsorte betrachtet 36 46-48

Peotta, Luana

kontrastive Fachtextpragmatik 26

176-184

Petelenz, Krzysztof

Online-Wörterbuch Polnisch-

Deutsch 28 231f

Pfister, Manfred

Intertextualität 16153

Philosophie 16 120f, 142

in der christlichen Ikonographie

16158

siècle philosophe 16125

Phonologie

Altniederländisch 10 129-131

Phraseme

Phrasem-Paare

äquivalente 3515

divergente 3515

heterovalente 3515

konvergente 3515

Phrasenprinzip 18 252, 2217

Phrasenstrukturgrammatik 24187

Phraseodidaktik 36 116-119

Phraseographie 36 91-120

Äquivalenzproblematik 36 108-116

Computer- 36 105f

dänisch-deutsche 3695

und Korpuslinguistik 36 97-102

und Lexikographie $3696 \mathrm{f}$

und Phraseodidaktik 36 116-119

theoretische 36 104-107

zwei- und mehrsprachige $36107 \mathrm{f}$

Phraseologie 35 267-273, 36 92-95, 37143

der Begriff 35 14, 271

dänisch-deutsche 3695

dänisch-isländische 3695

dänische 3695

deutsch-isländische 3695

Festigkeit 3535

formale Darstellung 35 12-14

formelhafte Texte 37143 
und geflügelte Worte 35250

Gegenstandsbereich 3525

als dritte Gliederungsebene der

Sprache 35 31f

isländische 3695

Konzeption

enge vs weite $3524 \mathrm{ff}$

österreichische 35 269-271

vs Parömiologie 35 29f, 3693

phraseologische Derivationen 35

83-94

Reduktion 35 89f, 92-94

Substitution 35 89-92

phraseologische Modifikationen

35 34f, 78ff, 83f

Modifikationsmechanismen 35

$83 \mathrm{ff}$

Typologie 3534

die phraseologische Normaldefinition 3514

Pumb aerobics- 35 271f

Sprachbestand 35 77-95

Spracherwerb $3535 f$

Stabilität vs Variabilität 35 77-83

Terminologie 35 14f, 28, 3694

im Text 35 32-35

auf Text-Ebene 35 30f

Textsortenabhängigkeit $3533 \mathrm{f}$

Umfang des Bereichs 35 24-32

in Wörterbüchern 36 102-107

Phraseologieforschung 36 94f

angelsächsische $\mathbf{3 5} 20$

dänisch-europäische 35 11-16

Dialekte $3536 \mathrm{f}$

Frühgeschichte 35 20-24

germanistische 35 17-39

Forschungslinien seit den 70er

Jahren 35 17-39

historische 3537

kontrastive interlinguale/intralinguale 3536

korpuslinguistische Analyse $3537 \mathrm{f}$

sowjetische 3524

Terminologie 3694

Uneinheitlichkeit 3538
Phraseologisierung 3583

Phraseologismen 36 91, 93 (Def)

Äquivalenz 36 108-116

Spiegelübersetzungen 36112

Austro- 35270

Doppelnatur der 36113

Einwort- 36 93f

Festigkeit 3525

pragmatische 35 30, 32f

Satz- 3529

semiotisches System $3531 \mathrm{f}$

Picht, Georg

Verantwortung 18177

Picht, Heribert

Fachsprachenforschung 22 175-189

Standortdefinition 22176

Terminologie 6 161-173, 21 181-

188

Pilz, Klaus D.

Phraseologie 3518

Pirttisaari, Pasi

Wortgruppenidiome 37143

PLAIN 1030

Plate, Ralf

Mittelhochdeutsches Wörterbuch

28231

Pöchhacker, Franz

Simultandolmetschen 14 272-275

Pörksen, Uwe

Textsorten 239

Poetik 23130

Pogner, Karl-Heinz

Schreibforschung 26 184-190

Polysemie 28212

in der Allgemeinsprache 28 211-221

in der juristischen Fachsprache $\mathbf{2 8}$

211-221

Poncela, Enrique J.

el humor 3346

Pors, Harald

Bedeutung 29 15f

Unterrichtsmaterial für Deutsch-

lerner 23 244-250

Portmann-Tselikas, P.R.

Übersetzung 33111 
Posteguillo, S.

wissenschaftliches Schreiben 22

184

Poulsen, Sven-Olaf

Textsortenkonventionen 967

Präpositionen

Ausdrucksselektion 17 193f

Inhaltsselektion 17192

vs Konjunktoren 17208

vs Subjunktoren $17207 \mathrm{f}$

Valenzeigenschaften 17 189-199

Valenzverhältnisse 17 181-209

Prawitz, Dag

Gebrauchstheorie 2981

Proformen

anaphorische 11 107-115

Projektcontrolling 757 (Fig)

Projektierung 7 53-68

Projektparameter 7 57f

Projektplanung 757 (Fig)

Proskription 25145

\section{Q}

Quadrivium 16124

Quak, Arend

Phonologie und Morphologie des

Altniederländischen 10 129-131

Quine, Willard V.

Bedeutungstheorie 2975

holistische Bedeutungskonzep-

tion 2982

Quintilian

körperliches Ausdrucksverhalten

23129

Rhetorik $23127 \mathrm{f}$

Qvistgaard, Jacques

Fachsprachenforschung 22 175-189

\section{$\mathbf{R}$}

Raible, Wolfgang

Fachlichkeit 16160

Rathmayr, Renate

interkulturelle Kommunikation
29233

Rechtslexikographie 35 255-258, $255 \mathrm{ff}$

Rechtsübersetzung 35 255-258,

259-265

Rechtsverständlichkeit

Leser-Text-Interaktion 29126

Probleme (Kommunikationsstö-

rung) 29 107-109

in der Schweizerischen Bundeskanzlei 29 111-122

zwischen Fachleuten und Laien im Ehescheidungsrecht 29 141161

Verbesserung von Gesetzestexten

29 118-120

Verständlichkeit von Rechtstexten

29 125-137

Rechtswissenschaft

vs Lexikographie $25256 \mathrm{f}$

vs Linguistik $25256 \mathrm{f}$

Recker, Ute

Mittelhochdeutsches Wörterbuch 28231

Referenten

Zugänglichkeitsgrad $1491 \mathrm{f}$

als Zugänglichkeitsmarkierer 14 91-94

Referenz

problematische 14 99-108

unproblematische 14 96-99

Reichmann, Oskar

Lexikographie 3145

Reiner, Arntz

Terminologie 6 161-173

Reiß, Katharina

AT-Analyse 3349

Ausgangs- und Zieltext 3379

Skopostheorie 3318 (Def)

Textäquivalenz 3350 (Def)

Textsortenkonventionen 9 67-69

Rektion $\rightarrow$ Kasusrektion;

Konjugationsartrektion

Rekurrenz 980

Reuter, Ewald 
Wirtschaftskommunikation 29232

Rhetorik 23 115-146

strukturelle Konstanten im rheto-

rischen System 23124

siehe auch accomodate dicere;

aptum; elocutio; sermo corporis;

virtutes

Richter, Gerd

das elektronische Flurnamenbuch

28231

Richter, Günther

Kommunikationstraining 874

Rickheit, Gert

kognitive Sprachverarbeitung 18

238-240

Rieger, Burghard

das Repräsentativitätsproblem

(Textkorpora) 6133

Robering, Klaus

Bedeutungstheorie 2917

Rojo López, A.M.

Humor 3352

institutional frames 3338

social frames $\mathbf{3 3} 36$

Rossenbeck, Klaus

Fachlexikographie 742

Rothkegel, Annely

Phraseologie 3518

Routineformeln 14 105, 3532

in Lernerwörterbüchern 37143

\section{S}

Sachbezogenheit 16 149f

Sachbuch 16145

Sache

die Sache im Zivilrecht 28 215-217

zur Terminologie der Sache im

Deutschen 28 211-221

das Tier als Sache $28217 f$

vertretbare und verbrauchbare

Sachen $\mathbf{2 8} 218 \mathrm{f}$

das Wort Sache in anderen

Rechtsgebieten 28 219-221

Sachlexikographie 755
Sachsprache 6129 (Def)

vs Fachsprache 6129

Sätze

deklarative 28 145-147, 150 f

exklamatorische 28 148f

imperativische 28 147f

Konditional- 28152

Struktur

Deutsch 24 206-209

Salager-Meyer, F.

Verweisstruktur in der medizini-

schen Literatur 22186

Sandberg, Bengt

es bei transitiven Verben 25 143-157

Sandrini, Peter

Übersetzertätigkeit 22183

Santana, Belén

Übersetzung humorvoller Literatur $3312 \mathrm{f}$

sapientia 16158

Sappler, P.

Bearbeitung eines Index 1033

Satzphraseologismen 3529

Satzsemantik

vs Wortsemantik 2982

Saussure, Ferdinand de Bedeutung eines Zeichens 2977

valeur linguistique 3347

Scenes 33 12, 15-41, 19 (Def), 51-

53,65

wesentliche Merkmale 33 21-24

Scenes-and-Frames 3311 (Def)

kognitive übersetzungsprozedurale

Konzeption 33 39-41

Terminologie des Konzepts 33 19f

in der Translationswissenschaft

33 15-41

für den Übersetzungsunterricht

33 15-41

Scharnhorst, Jürgen

Sprachkultur 37135 (Def)

Wörterbuchbenutzerforschung 37

$131 \mathrm{f}$

Schendera, Christian

Rechtsverständlichkeit 29108 
Schlaefer, Michael

Wörterbuchbenutzung 36 118f

Schmeisser, Wilhelm

internationales Management 29239

Schmidt, Ingrid

Publikationsmodell in der Lexi-

kographie 28 224f

Schmitz, K.-D.

rechnergestützte

Terminologieverwaltung 22182

Schoser, Franz

Wirtschaftskommunikation 29 224f

Schreiben

wissenschaftliches 22184

Schreibforschung 26 184-190

Schröder, Bernhard

computergestützte Lexikographie 28 223-235

Schröder, Hartmut

fremdkulturelles Textverstehen

29235

Schulz von Thun, Friedemann

empirisch-induktives

Verständlichkeitskonzept 16140

Kommunikationsmodell 29143

Science-fiction 33 15-41

scientia 16157

Scripts 33 33-35, 33 (Def)

Selnow, Gary W.

Wirtschaftskommunikation $29237 \mathrm{f}$

Semantik 29 87-93

bedeutungstheoretische Prinzipien

29 94-97

Bivalenzprinzip $2995 \mathrm{f}$

semi-strukturelle 2995

gebrauchstheoretische Fundierung 29 99-103

Gebrauchstheorie der Bedeutung

29 73-103

referenztheoretische Fundierung

29 97-99

Regeln 29 88-90

Regelwerke 29 91-93

Rolle der Bedeutungstheorien 29

93-99
Satz-

vs Wortsemantik 2982

semantische Beschreibungen 29

13-20

semantische Netze 38 154, 166

semantische Spiele 2983

Wort-

vs Satzsemantik 2982

semasiologische Prinzip, das

vs das onomasiologische Prinzip

6168

Seme 6115

Sememe 6115

Archi- 6116

Semiotik

fachkommunikative 16152

Semiotizität 16152

Sententialisierung 33109

sermo corporis 23129

SGML 28224

Sialm, Ambros

Phraseologie 35 18, 24

Terminologie 3528

pragmatische Phraseologismen 35

32

Typologie der Modifikationen 3534

siècle philosophe 16125

Signalfunktion 980

Signalwirkung 980

Simultandolmetschen 11 107-115,

14 89-109, 272-275

Diskursmodelle 14 90f

dolmetschrelevante Parameter 19

$285 f$

Fremdbestimmtheit 1490

als Gegenstand der interdiszipli-

nären Forschung 19 282-288

zur Geschichte 19283

und kognitionspsychologische

Forschung 19285

und neurophysiologische Forschung

$19287 f$

Persönlichkeitsuntersuchungen an

Dolmetschern 19 286f

pronominale Wiederaufnahme $\mathbf{1 1}$ 
109-115

Qualität der Dolmetschleistung $19283 f$

Verzögerungsstrategie 11 113f

Wiederholung des Ausgangs-

diskurses 3377

Zugänglichkeitsmarkierer 14 91-

94, $98 \mathrm{ff}$

Unterschiede in der Anwendung

14 96-108

Sionis, Claude

Fachsprachenforschung $22179 f$

Situationen $14272 f$

Typen 23236

Skopostheorie 3318 (Def)

Skyum-Nielsen, P.

Fachsprachenforschung 22187

Snell-Hornby, Mary

erste Herausforderung des Über-

setzers 3352

Funktion einer litterarischen

Übersetzung 3362

Mehrdimensionalität des komischen

Phänomens 33 52f

Scenes 3319

and Frames 33 20, 23

Sønderjysk 24 183f

Solfjeld, Kåre

Sententialisierung 33109

Substantivrahmen 2225

Sophía 16 118-121, 126

Sowinski, B.

Textsorten 245

Spanisch

Vornamengebung

in der spanischsprachigen Welt 3356

Sperber, Dan

menschliche Kognition 970

Spiegelübersetzungen 36112

Spillner, Bernd

Ghostwriter 875

Sprachberatung

und Wörterbuchbenutzung $37132 \mathrm{f}$

Sprache 23 123f
Allgemein- 28215 (Def)

Alltags- 28215 (Def)

Begegnungs- 16164

Erschließungs- 16164

Fundamental- 16164

gesprochene

Dänisch vs Deutsch 23 233-238

Körper-

in Wirtschaftskommunikation

23 115-146

Sach- 6129 (Def)

vs Fachsprache 6129

Umgangs- 28215 (Def)

Verkehrs- 16164

Wirtschafts- 29220

Substantivphrase in deutschen

Wirtschaftstexten 18 251-254

siehe auch Fachsprache

Spracherwerb

als Forschungsbereich $3535 f$

Sprachfunktionen 23235

responsorientierte Sprachfunktion

23235

Sprachkultur 16 156, 37 131-136,

135 (Def)

Sprachlexikographie 755

sprachliche Handlungen 9 72-76

sprachliche Merkmale

Kodierungsgrad 9 68-81

Signalfunktionen 9 68-81

Sprachpolitik 16164

Sprachplanung auf Island 22186

Sprachspiele 29 83-87, 90f, 3535

W-spiel

in gebrauchstheoretischer Sicht

29 100-102

Sprachwissenschaft $\rightarrow$ Linguistik

Sprichwörter 35 29f

Anti- $\mathbf{3 5} 30$

japanische $35272 \mathrm{f}$

und kognitive Metapherntheorie

$35268 \mathrm{f}$

und Wendungen 3581

Stein, Stephan

Phraseologie auf Text-Ebene 3530 
pragmatische Phraseologismen 35 32

Stephan, Peter

deutsche Satzstruktur 24 206-209

Stilistik

kognitiver Stil 879

sprachliche Zweifelsfälle 8 55-69

Wissenschaftssprachstilistik 16 140f

Stimulus-Response-Muster 2977

Stock, Richard

Präsentationstechnik 29240

Stötzel, Georg

kontroverse Begriffe 16130

Stolze, Radegundis

hermeneutisches Übersetzen 10

109-114

Texte 3349

Storrer, Angelika

computergestützte Lexikographie

28 223-235

digitale Wörterbücher $28225 f$

Strauß, Gerhard

brisante Wörter 16130

schwere Wörter 16130

Strohner, Hans

kognitive Sprachverarbeitung 18

238-240

Strother, Judith B.

interkulturelle Kommunikation

22185

Stubkjær, Flemming T.

Unterrichtsmaterial für Deutsch-

lerner 23 244-250

Subjunktionen 17200

Subjunktoren

Ausdrucksvalenz 17200

Null- 17200

vs Präpositionen $17207 f$

Valenzeigenschaften 17 200-204

Valenzverhältnisse 17 181-209

Substantivphrasen

in deutschen Wirtschaftstexten 18

251-254, 22 13-46

Typen von 22 43-45

Substantivrahmen 22 18, 25
Substitution, lexikalische $3537 f$

Südschleswigdänisch 24182

SUSY 1030

Synonymie 18 138, 28212

Syntax

dependentiell 24 185-189

Deutsch 24 185-189

deutsche Satzstruktur 24 206-209

Generative 24186

siehe auch Drehsatz; Wortstellung

Systematizität 16150

\section{$\mathbf{T}$}

Tarp, Sven

bilinguale Wörterbücher $37140 f$

die moderne lexikographische

Funktionslehre

vs die allgemeine Theorie der

Lexikographie 38 141f

Wörterbuchfunktionen 37139

Tausch, Reinhard

empirisch-induktives

Verständlichkeitskonzept 16140

téchnai enkyklioi 16124

téchne 16 119-121

Technical Writing 16 141f

Technisches Schreiben $16141 \mathrm{f}$

Teich, Elke

Übersetzung 33111

Tel Quel 16153

Tene, A. Ndeffo

Übersetzung von hybriden littera-

rischen Texten 3313

Terborg, Heiner

Deutsch als Fremdsprache 8 80f

Term

der Begriff 22181

polyseme Termini $22182 \mathrm{f}$

Vertextung von Termini 23 41-62

TERMDOK

Rezension 18 241-245

Terminographie

vs Fachlexikographie 6 167f

Terminologie 6 161-173, 7 53-68, 
21 181-188

begriffliche Strukturierungen 18

33-49

Benennungen 6166

vs Fachsprache 6162

Forschung $22181 \mathrm{f}$

vs Lexikographie 4 75, 21 185f

Normung 6166

Studium 6172

systematische Begriffsrepräsentation $\mathbf{1 8} 46 \mathrm{f}$

terminologische Sphären 6166 terminologisches Methodenwissen $1842 \mathrm{f}$

Vertextung von Termini 23 41-62

Wissensorganisation und 22 153158

Terminologiewissenschaft $\rightarrow$ Terminologie

Text-Wortformen $\mathbf{4} 75$

vs Lexikon-Wortformen 3581

Textäquivalenz 3350 (Def)

Textbereich 237 (Def)

Texte 14 273, 29 128-131, 33 49,

76 (Def)

der Begriff 29 134f, 33 75f

Fach-

juristische

Notwendigkeit der Komplexität 3661

formelhafte 3530

Intertextualität 16 153, 33 64f

Leser-Text-Interaktion 29 127-135

Leser-Text-Modell 29134

Parallel- 3365 (Def)

Primär- vs Sekundär- 1489

vorgeformte 3530

Textgattung 237 (Def)

Textgrammatik 17 133f

Textgruppe 237 (Def)

Textklasse 237 (Def)

Textklassifikation 2 37ff

Textkorpora

dänisch und deutsch für Gentechnik 6 125-137
Erstellung durch maschinelle

Unterstützung 5101

Korpus vs Sprachsystem $6133 \mathrm{f}$

Phraseologismen in 36 97-102

Frequenzbestimmungen 36 98-

100

das Repräsentativitätsproblem 6

132-134, 35 37, 264

das Statusproblem 6132

Überprüfung lexikographischer

Informationen 36 100-102

Textlinguistik 16 139, 3671

Fach- 13 326-330

integrative 18 203-205

Textologie 22159 (Def)

Textproduktion

sekundäre vs primäre 14 89-91

Textsinn 3349

Textsorten 2 37-53, 9 69, 35 31-34

die Anzeige 244

der Begriff 2 38-42, 39 (Def)

die Durchführungsbestimmung 242

einfache 2 40f

Fach-

im Englischen 8 93-98

der Fachzeitschriftenartikel 23

51-53, 58-62

die Inhaltsangabe 241

als kodierte Elemente 9 76-80

und Kommunikation 2 46-48

komplementäre 241

komplexe 240

das Lehrbuch 23 51, 58-61

das Lexikon 23 51, 60f

in Naturwissenschaften und

Technik 16228

die Parodie 242

das Patent 36 46-48

der populärwissenschaftliche

Zeitschriftenartikel 23 51f, 59-62

das Protokoll 2 45f

die Rezension 241

und Sprachgemeinschaft 2 48f

technische 23 41-62

Stellenwert des Adressaten 23 
41-49

die Travestie 242

Textsortenklassen 2 42-46

einzelsprachliche 248

Konventionen in 2 44-46

übereinzelsprachliche $248 \mathrm{f}$

universale 2 48f

Textsortenklassifizierung 238

Textsortenkonventionen 2 37-53, 9

67-70, $16233 f$

und Äquivalenzkriterien $251 \mathrm{f}$

als Auslöser von Erwartungshal-

tungen $246 \mathrm{f}$

als Erkennungssignale 246

Gerichtsurteile

Deutsch und Dänisch 22 158-160

als Steuerungssignal für das

Textverstehen 2 47f

und Übersetzung 2 48-52

Textsortentypologie 16 231-233

Textsortenvarianten

Konventionen in 2 44-46

Texttypen 237 (Def), 50f, $893 \mathrm{f}$

Theatralik 23 115-146

Thesaurus 6 170f

Third-Party-Kante 38163

Thomsen, H.E.

Terminologieforschung 22181

Thun, Harald

Phraseologie 3519

Tiittula, Liisa

internationale Geschäftskommu-

nikation 29232

internationale Geschäftsverhand-

lungen $29229 f$

Togeby, Ole

Bedeutung 2918

die Modalpartikel nok 28150

Topic-Map-Standard 38154

Topic-Maps 38154

translatio studii 16125

Translation Studies, Descriptive 3362

Traversierung 38159 (Def)

Trivium 16124

Tysk-tysk ordbog (Munk)
Rezension 6157

$\mathbf{U}$

Übersetzung 2 37-53, 8 81-84, 9

68f, 10 109-114

Äquivalenzkriterien 3 169-175

Attribute

deutsche

Wiedergabe in norwegischen

Übersetzungen 33 89-112

Präpositional- 33 106f

der Begriff

und translatorische Relevanz 33

76-78

Bibel- 3372

Didaktik 33 71-86

Empfänger 3325

expliziter als das Original 33 91f

Explizitheit 3392

hermeneutisches 10 109-114

Humor 33 45-67

humorvoller Literatur 33 12f

interkultureller Transfer 33 50-53

das Komische 3350

Komposita 33 97f

litterarische 33 9-13

Funktion 3362

kulturspezifische Elemente $3363 \mathrm{f}$

Mittler 3325

nachgestellte Partizipialkonstruk-

tionen 33106

Nominalisierungen 33 107f

norwegische

Wiedergabe deutscher erweiterter

Attribute 33 89-112

Präpositionalattribute 33 106f

Praxis 16147

prospektive und retrospektive $\mathbf{3 3} 72$

Rechts- 35 255-258, 259-265

Relativsätze 33 105f

Satzumformungen 33108

Scenes-and-Frames in 33 15-41

Sender 3325

Sinn 110 
Spiegel- 36112

sprachliche Handlungen 970

Stimmigkeit $10110 \mathrm{f}$

Strategien 3393

und Textsortenkonventionen 2

48-52

und Äquivalenzkriterien 2 51f

Theorie 882

Tilgung

Attribute 33 101f

Erweiterungen 33 99-101

Translationsregeln 16 234f

translatorische Kategorien 10 109f,

112

translatorische Relevanz 33 71-86,

77 (Def)

und Adäquatheit 33 78f

als didaktisches Problem 33 79-84

theoretische Status 33 74-79

und Übersetzungsstrategien 33

$71-86$

Typen $250 \mathrm{f}$

Interlinearversion 3162

kommunikative $3162 \mathrm{f}$

philologische 3162

wörtliche 3162

Typen von Umsetzung 882

der Übersetzer 33 15-41

Aufgabe 33 65-67

Doppelrolle $3351 \mathrm{f}$

Fertigkeit 10114

Tätigkeit $22183 \mathrm{f}$

Zielstrukturen

analoge 33 94-97

fehlende 33 102-104

siehe auch Adäquatheit; Äquiva-

lenz; Translation Studies, De-

scriptive; Dolmetschen; Simul-

tandolmetschen

Übersetzungswissenschaft 10 109f

vs Sprachwissenschaft 10109

Uhl, Gerhard

Business-Etikette in Europa 34

285-292

Uhl-Vetter, Elke
Business-Etikette in Europa 34

285-292

Umgangsformen 34 285-292

Umgangssprache 28215 (Def)

Unger, Thorsten

Lachkultur 3346

Unwissenschaftlichkeit

vs Wissenschaftlichkeit 35240

Urteile 28 88-90

V

Valenz

-Eigenschaften 17182

bei Präpositionen 17 189-199

bei Subjunktoren 17 200-204

-Verhältnisse bei Präpositionen,

Subjunktoren und Konjunktoren

17 181-209

Ausdruck $17183 \mathrm{ff}$

Ausdrucks- 17188

der Begriff 17 181f

Dependenz 17 184ff

Inhalt $17183 \mathrm{ff}$

Inhalts- 17188

Partikel-

im Deutschen 17 181-209

valeur 3347

valeur linguistique 3347

Vannerem, Mia

Scenes 3319

and Frames 33 20, 23

Variantenwörterbuch der

deutschen Sprache 3536

Vega, Manuel de

Frame-Typologie 3327

social frames $\mathbf{3 3} 36$

Verben

Äußerungs-

Dänisch vs Deutsch 9 74-79

es bei transitiven 25 143-157

verbotene Künste 16125

Verhandlungen

die mündliche Gerichtsverhandlung 22 133-148 
Vermeer, Hans J.

Scenes 33 19f

Skopostheorie 3318 (Def)

Vernetzungen (lexikographisch) 38

146f (Def)

bidirektionale 38149 (Def), 167

(Def)

Datenbasis-externe 38 148f

Datenbasis-interne 38 148f, 151

Inhaltsstruktur-interne 38 147, 151

unidirektionale 38 149ff, 150

(Fig), 158 (Fig), 167 (Def)

Vernetzungsstruktur (lexikographisch) 38 137-168

vs Inhaltsstruktur 38148

vs Mediostruktur 38 145f

Verständlichkeitskonzept, empi-

risch-induktives 16140

Vertextung 23 45f (Def)

Verweisstruktur (lexikographisch)

38137

Viehweger, Dieter

Situationstypen 23236

Vikner, Sten

deutsche Satzstruktur 24 206-209

da Vinci

Leonardo

Körperausdruck 23117

Vinogradov, V.V.

Phraseologie 3524

virtutes 23138

Vogel-Jørgensen, T.

geflügelte Worte 35243

Vornamengebung

in der spanischsprachigen Welt 3356

\section{W}

Wagner, Andreas

GermaNet $28232 \mathrm{f}$

Wagner, Johannes

kommunikative Barrieren $29231 \mathrm{f}$

WAHRIG $\rightarrow$ Deutsches Wörter-

buch (WAHRIG)
Wahrman, S.N.

Wörterbücher 7 34, 41

Wandruszka, Mario

Übersetzungen sind expliziter als

das Original 3391

Wawrzyniak, Zdzislaw

Übersetzung 8 81f

Weinrich, Harald

Lexikographie 18178

Sprachpolitik 16164

Subjunktoren/Konjunktoren 17203

Welter, Rüdiger

Goethes Wortschatz und das

Goethe-Wörterbuch 28 229f

Wendungen

und Sprichwörter 3581

Wengeler, Martin

kontroverse Begriffe 16130

Wessela, Eva

Wirtschaftskommunikation 29

222f, 234

Weydt, Harald

Definition der MP-Funktion 28137

Fremdsprachenerwerb 17 170-172

Übersetzung 8 83f

Wiegand, H.E.

allgemeine Theorie der Lexiko-

graphie

vs die moderne lexikographische

Funktionslehre 38 141f

lexikographische Daten 38 142f

die lexikographische Definition 3

144

Verweise 38146

Beziehungen 38147

Verweisvoraussetzungen 38143

Wijst, Per van der

Höflichkeitsstrategien 29230

Wikforss, Jonas

Wörterbücher 7 34, 40f

WIKO-Modell 22154

Wills, Wolfram

Übersetzungsfertigkeit 10114

Sekundärtexte vs Primärtexte 1489

Wilson, Deirdre 
menschliche Kognition 970

Winge, Vibeke

deutsche Einwanderergruppen in

Dänemark 6 195f

Wirtschaftskommunikation 29217 242

Adaption 29240

Informationstechnologien 29 236239

interkulturelle 23 115-146

interne $29234 \mathrm{f}$

Körpersprache in 23 115-146

Lakunen 29235 (def), 235f

und Marketingkommunikation 29

219-221

in Osteuropa 29 232-234

Stereotypen $29231 \mathrm{f}$

siehe auch Marketingkommunikation

Wirtschaftslinguistik 23135

Wirtschaftssprache 29220

Substantivphrase in deutschen

Wirtschaftstexten 18 251-254

siehe auch Fachsprache

Wissen 18 41-43, 3310

Fach- 1842

vs Information 22155

Wissensbasis 759

Wissenschaft 16120

der Begriff 16157

scientia 16157

Wissenschaftlichkeit

vs Unwissenschaftlichkeit 35240

Wissenschaftsjournalismus 16165

Wissensorganisation

und Terminologie 22 153-158

Wissenssysteme 33 9-13

kulturbezogene

Relation zu literarischen Texten 33 9-13

Theorie der 3311 (Def)

Witte, Heidrun

Scenes 33 19f

Wittgenstein, Ludwig

Gebrauchstheorie 2974 illokutionäre Kraft und propositionales Gehalt einer Äußerung

2984

Sprachspiele 29 84, 86, $90 \mathrm{f}$

Wöllstein-Leisten, A.

deutsche Satzstruktur 24 206-209

Wörterbuch der deutschen Gegenwartssprache 3 148ff

vgl. mit den Duden-Bänden 8 59ff

Wörterbuch der österreichischen

Phraseologie 35 269-271

Wörterbuch Deutsch als Fremdsprache (de Gruyter)

Rezension 25 159-168

Wörterbuch-Wortformen 475

Wörterbücher(n)

aktive 4 72f, 6 111, 120, 37140

Angaben

der Terminus 38140

Vor- 38140

Benutzerdiskurs 21211 (Def)

Benutzer(gruppen) 6 169, 37145

das Benutzerprofil 21211

Benutzerintentionen 21211

Benutzermatrix

Positionen in der 21211

Benutzersituationen 21211

Benutzerstruktur 21211 (Def)

Benutzervoraussetzungen 21211

Benutzung 36 118f, 37 131-136

und Sprachberatung $37132 \mathrm{f}$

Benutzungsanleitungen $37141 \mathrm{f}$

Didaktik 36119

einsprachige 37 139-148

Hauptaufgabe 3 143f

elektronische

fachlexikographische Maßstäbe

für 18 241-245

Fach- 4 67ff, 7 29-50

Beispiele in $743 \mathrm{f}$

Belege in $743 \mathrm{f}$

Benutzung 4 68-78, 81 (Fig)

bidirektionale $748 \mathrm{f}$

eigene Bildungen in $747 \mathrm{f}$

ein- vs zweisprachige 743 
enzyklopädische Glossen 7 43, 49 enzyklopädische Informationen in 7 41-44, 21 205-213 falsche Freunde in 747 Grammatik in $744 f$ historischer Wortschatz in $738 \mathrm{f}$ als Kontrollinstanz 736 mehrsprachige 7 48-50 Mehrworttermini in 737 Metasprache in $745 \mathrm{f}$ multidirektionale 7 48-50

Neologismen in 738 Phraseologie in $740 \mathrm{f}$ Prinzip der Verifizierung 747 semantische Informationen in 21 205-213

Stichwortauswahl in 7 33-40 unidirektionale $748 \mathrm{f}$

Vollständigkeit in 7 34-36

Wortbildung in 7 35-38

Zuverlässigkeit der Angaben in 7 46-48

Funktionen 37139

Kern- 37 139, 146f

Korrektur 37142

wissensbezogene 37 146f

Dokumentation 37146

Lemma

-Lücken 37143

-Selektion 37 143f

Lemmatisierung 6114

Probleme 10 34-38

Repräsentationsprobleme 1033 vs Wort 6114

Lerner- 25 159-168 und Grammatik 878

Lexemwissen repräsentatives und ergänzendes $21211 f$

Lexika als Textsorte betrachtet 23

51

das lexikographische Nachschlagewerk $21209 f$

passive 4 72f, 6 120, 37140

Phraseologie in 36 102-107
Strukturen

Inhalts- 38 140f

vs Vernetzungsstrukturen 38148

Makro- 7 33-40

Medio-

vs Vernetzungsstrukturen $\mathbf{3 8}$ $145 \mathrm{f}$

Mikro- 7 40f

Vernetzungs- 38 137-168

vs Inhaltsstrukturen 38148

XML-basierte Modellierung

38 137-168

Verweis- 38137

synonymische Beziehungen 3

143-158

Theorie und Empirie $21207 \mathrm{f}$

Typologie 21210

Kritik der Wiegand'schen

Auffassung 35264

Verweise 38146

Beziehungen 38147

im elektronischen Wörterbuch?

38143

Verweisvoraussetzungen 38143

Wörterbuchgrammatik 6 141-143

Wörterbuchkriminalität 6157

in der Zukunft 37 133-135

zweisprachige 6 111-122, 37 139148

äkvivalentlose Lemmata in 6 111-122

kulturgebundene Wörter in 6

111-122

Ortographie $37142 \mathrm{f}$

siehe auch Lexikographie; Lexikon-Wortformen; Text-Wortformen; Wörterbuch-Wortformen

Wolf, Christa 6199

Wolf-Bleiß, Birgit

Sprachberatung und

Wörterbuchbenutzung 37 132f

Wort

Wörter vs Lemmata 6114

Wortformen

Lexikon- 475 
vs Text-Wortformen 3581

Text- 475

Wörterbuch- 475

Wortgruppenidiome 37143

Wortschatz

synonymische Beziehungen 3

143-158

Wortsemantik

vs Satzsemantik 2982

Wortstellung

Deutsch vs Polnisch 879

Wortverbindungen

feste

unstabile 35 77-95

freie 3525,31

Wotjak, Barbara

Routineformeln in Lernerwörter-

büchern 37143

die Textsortenabhängigkeit der

Phraseologie 3334

Wendungen und Sprichwörter 3581

Wroblewski, Jerzy

Rechtsverständlichkeit 29135

Wüster, Eugen

begriffliche Strukturierungen 1834

Terminologie 6 163, 165

\section{$\mathbf{X}$}

XLink 38 153-155

XML 28224

-Modellierung

Vernetzungsstrukturen

lexikographischer Daten 38

137-168

\section{$\mathbf{Z}$}

Zieltext 33 71ff

Zifonun, Gisela

schwere Wörter 16130

Zitate

vs geflügelte Worte 35 245-248

Zitathaftigkeit 35 246-248

Zuluaga, Alberto
Phraseologie 3520

Zwillingsformeln 3527 
